\title{
Heat Transfer and Mass Diffusion in Nanofluids over a Moving Permeable Convective Surface
}

\author{
Muhammad Qasim, ${ }^{1}$ Ilyas Khan, ${ }^{2,3}$ and Sharidan Shafie ${ }^{2}$ \\ ${ }^{1}$ Department of Mathematics, COMSATS Institute of Information Technology, Park Road, Chak Shahzad, Islamabad 44000, Pakistan \\ ${ }^{2}$ Department of Mathematical Sciences, Faculty of Science, Universiti Teknologi Malaysia (UTM), Johor Barhu, 81310 Skudai, Malaysia \\ ${ }^{3}$ College of Engineering Majmaah University, P.O. Box 66, Majmaah 11952, Saudi Arabia
}

Correspondence should be addressed to Ilyas Khan; ilyaskhanqau@yahoo.com

Received 14 July 2013; Revised 13 September 2013; Accepted 13 September 2013

Academic Editor: Waqar Khan

Copyright (c) 2013 Muhammad Qasim et al. This is an open access article distributed under the Creative Commons Attribution License, which permits unrestricted use, distribution, and reproduction in any medium, provided the original work is properly cited.

\begin{abstract}
Heat transfer and mass diffusion in nanofluid over a permeable moving surface are investigated. The surface exhibits convective boundary conditions and constant mass diffusion. Effects of Brownian motion and thermophoresis are considered. The resulting partial differential equations are reduced into coupled nonlinear ordinary differential equations using suitable transformations. Shooting technique is implemented for the numerical solution. Velocity, temperature, and concentration profiles are analyzed for different key parameters entering into the problem. Performed comparative study shows an excellent agreement with the previous analysis.
\end{abstract}

\section{Introduction}

Usually, the conventional heat transfer fluids such as oil, water, and ethylene glycol mixtures are poor heat transfer fluids because of their poor thermal conductivity. Therefore, several attempts have been made by many researchers to enhance the thermal conductivity of these fluids by suspending nano/microparticles in liquids. Amongst them Choi [1] was the first who introduced a fluid with enhanced thermal conductivity known as nanofluid. At present, the flow problem involving nanofluids has attracted the investigators to the field. These fluids are engineered colloidal suspensions of nanoparticles (nanometer-sized particles of metals, oxides, nitrides, carbides, or nanotubes) in the ordinary base fluid. Thermal conductivity of nanofluids is higher than the base fluids. Such fluids over a moving surface with heat transfer seem to be very important in microelectronics, fuel cells, hybrid-powered engines, and pharmaceutical processes. It should be pointed out that several metallurgical processes involve the cooling of continuous strips or filaments by drawing them through a nanofluid [2]. Having that in mind, the boundary layer flows of nanofluids have been studied through different approaches, in the recent attempts [3-15] there has been an increasing interest of the recent researchers in the studies of boundary layer flows over a moving surface with heat transfer. This interest in fact stems from several applications of such flows in aerodynamic extrusion process, paper production, food process, glass fiber production, metallurgical process, and so forth. Sakiadis [16] made an initial attempt for boundary layer flow induced by a continuous solid surface moving with constant speed. Tsou et al. [17] extended the work of Sakiadis [16] to heat transfer concept in the boundary layer flow on a continuous moving surface. Abdelhafez [18] studied the boundary layer flow over a continuous moving flat surface in a parallel free stream. He studied the case when surface and free stream move in the same direction. Afzal et al. [19] revisited similar problem and reported the case when the surface and free stream move in the opposite directions. Ishak et al. [20] extended the work of Afzal et al. [19] by considering viscous dissipation and mass transfer effects. They investigated both the cases when surface and free stream move in the same direction and opposite directions. They obtained the numerical solution of the problem by a finite difference scheme known as Keller 


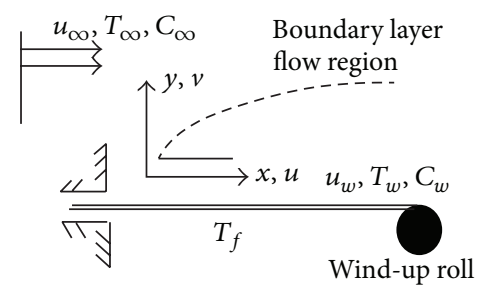

Figure 1: Physical flow model.

box method. Very recently, Aziz [21] obtained a similarity solution for Blasius flow of a viscous fluid employing convective boundary conditions. Hayat et al. [22] investigated the flow of Maxwell fluid over a stretching sheet with convective boundary conditions. Some more interesting problems with convective boundary conditions have been reported [23-29].

The present paper concentrates on the numerical study of the boundary layer flow of a nanofluid over a permeable moving surface. Mathematical model is constructed in the presence of Brownian and thermophoresis effects. Governing nonlinear analysis is computed by shooting method. Results are compared and analyzed in detail.

\section{Problem Statement}

We study flow of nanofluid over a moving permeable surface with constant velocity $u_{w}$ in the parallel direction of the uniform free stream velocity $u_{\infty}$. The constant temperature and concentration of wall are, $T_{w}$ and $C_{w}$, respectively. The ambient values of temperature and concentration are respectively, $T_{\infty}$ and $C_{\infty}$. In Cartesian coordinate system, $x$ - and $y$-axes are chosen parallel and perpendicular to the moving surface (see Figure 1).

The boundary layer equations (in absence of viscous dissipation) for the physical problems under examination are

$$
\begin{gathered}
\frac{\partial u}{\partial x}+\frac{\partial v}{\partial y}=0 \\
u \frac{\partial u}{\partial x}+v \frac{\partial u}{\partial y}=-\frac{1}{\rho_{f}} \frac{\partial p}{\partial x}+\nu \frac{\partial^{2} u}{\partial y^{2}} \\
u \frac{\partial T}{\partial x}+v \frac{\partial T}{\partial z}=\alpha \frac{\partial^{2} T}{\partial y^{2}}+\tau\left[D_{B}\left(\frac{\partial C}{\partial y} \frac{\partial T}{\partial y}\right)+\frac{D_{T}}{T_{\infty}}\left(\frac{\partial T}{\partial y}\right)^{2}\right] \\
u \frac{\partial C}{\partial x}+v \frac{\partial C}{\partial z}=D_{B} \frac{\partial^{2} C}{\partial y^{2}}+\frac{D_{T}}{T_{\infty}} \frac{\partial^{2} T}{\partial y^{2}}
\end{gathered}
$$

where $u$ and $v$ are the components of the velocity along the $x$-and $y$-directions, respectively, $\rho_{f}$ is the density of the base fluid, $v\left(=\mu / \rho_{f}\right)$ is the kinematic viscosity, $\alpha$ is the thermal diffusivity, $D_{B}$ is the Brownian motion coefficient, $D_{T}$ is the thermophoretic diffusion coefficient, and $\tau=\left((\rho c)_{p} /(\rho c)_{f}\right)$ is the ratio of effective heat capacity of the nanoparticle material to the heat capacity of the fluid.
The boundary conditions in view of physics of the present problem are

$$
\begin{gathered}
u=u_{w}, \quad v=v_{w}, \\
-k \frac{\partial T}{\partial y}=h_{f}\left(T_{f}-T\right), \quad C=C_{w} \quad \text { at } y=0, \\
u \longrightarrow u_{\infty}, \quad T \longrightarrow T_{\infty}, \\
C \longrightarrow C_{\infty} \quad \text { as } y \longrightarrow \infty .
\end{gathered}
$$

It seems worth mentioning to point out that $u_{w}=0$ corresponds to the Blasius problem and for $u_{\infty}=0$ we have the Sakiadis problem. Here $h$ is the heat transfer coefficient and $T_{f}$ is the convective fluid temperature below the moving surface. A stream of cold fluid at temperature $T_{\infty}$ moving over the right surface of the plate with uniform velocity $u_{\infty}$ while the left surface of the plate is heated below by the convection from the hot fluid of temperature $T_{f}$ which provides a heat transfer coefficient $h_{f}$. As a result, convective boundary conditions arise.

We define the dimensionless quantities given by

$$
\begin{gathered}
\psi=\sqrt{x \nu U} f(\eta), \quad \theta(\eta)=\frac{T-T_{\infty}}{T_{f}-T_{\infty}}, \\
\phi(\eta)=\frac{C-C_{\infty}}{C_{w}-C_{\infty}}, \quad \eta=\sqrt{\frac{U}{x \nu}} y,
\end{gathered}
$$

where $U=u_{w}+u_{\infty}$, and the free stream function $\psi$ satisfies

$$
u=\frac{\partial \psi}{\partial y}, \quad v=-\frac{\partial \psi}{\partial x} .
$$

The above expression also satisfies the continuity equation (1) and (2)-(5) are reduced to the following forms:

$$
\begin{gathered}
f^{\prime \prime \prime}+\frac{1}{2} f f^{\prime \prime}=0, \\
\theta^{\prime \prime}+\frac{\operatorname{Pr}}{2} f \theta^{\prime}+\operatorname{Pr} N_{b} \theta^{\prime} \phi^{\prime}+\operatorname{Pr} N_{t} \theta^{\prime 2}=0, \\
\phi^{\prime \prime}+\frac{\operatorname{Le}}{2} f \phi^{\prime}+\frac{N_{t}}{N_{b}} \theta^{\prime \prime}=0, \\
f(0)=S, \quad f^{\prime}(0)=\epsilon, \quad f^{\prime}(\infty)=1-\epsilon, \\
\theta^{\prime}(0)=-\gamma(1-\theta(0)), \quad \theta(\infty) \longrightarrow 0, \\
\phi(0)=1, \quad \phi(\infty)=0 .
\end{gathered}
$$

Here primes denote differentiation with respect to $\eta, f(0)=S$ with $S>0$ corresponding to suction case and $S<0$ implying injection, $\operatorname{Pr}(=v / \alpha)$ is the Prandtl number, Le $\left(=v / D_{B}\right)$ is the Lewis number, $N_{b}\left(=(\rho c)_{p} D_{B}\left(C_{w}-C_{\infty}\right) /(\rho c)_{f} \nu\right)$ is the Brownian motion parameter, $N_{t}\left(=(\rho c)_{p} D_{T}\left(T_{f}-\right.\right.$ $\left.\left.T_{\infty}\right) /(\rho c)_{f} T_{\infty} \nu\right)$ is the thermophoresis parameter, $\gamma=\left(h_{f} /\right.$ $k \sqrt{v x / U})$ is the Biot number, and $\epsilon\left(=u_{w} / U\right)$ is the velocity ratio parameter. Further we noticed that $\epsilon=0$ corresponds 


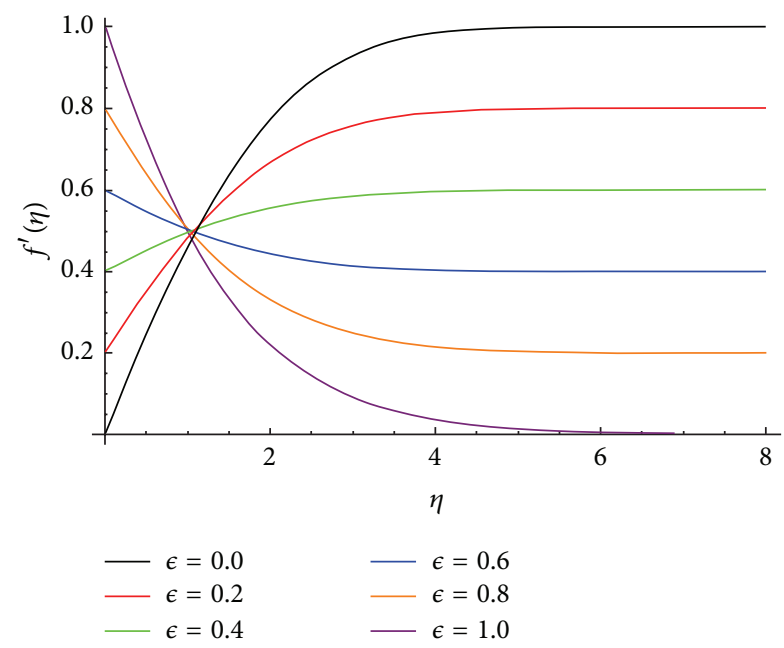

FIGURE 2: Velocity profile $f^{\prime}(\eta)$ for various values of $\epsilon$ when $S=1.0$.

to the flow over a stationary surface caused by the free stream velocity while $\epsilon=1$ is subjected to a moving plate in an ambient fluid, respectively. The case $0<\epsilon<1$ holds when the plate and fluid are moving in the same direction. If $\epsilon<0$, the free stream is directed towards the positive $x$-direction, while the plate moves towards negative $x$-direction. On the other hand, if $\epsilon>1$, the free stream is directed towards negative $x$-direction, while the plate moves towards the positive $x$ direction. Here we only discussed the case when $0 \leq \epsilon \leq 1$.

Expressions for the local Nusselt number $\mathrm{Nu}_{x}$ and the local Sherwood number $\mathrm{Sh}_{x}$ are

$$
\begin{gathered}
\mathrm{Nu}_{x}=\frac{x q_{w}}{k\left(T_{w}-T_{\infty}\right)}, \\
\mathrm{Sh}_{x}=\frac{x j_{w}}{D_{B}\left(C_{w}-C_{\infty}\right)},
\end{gathered}
$$

where the wall heat flux $q_{w}$ and the mass flux $j_{w}$ are given by

$$
\begin{aligned}
& q_{w}=-k\left(\frac{\partial T}{\partial y}\right)_{y=0}, \\
& j_{w}=-D_{e}\left(\frac{\partial C}{\partial y}\right)_{y=0} .
\end{aligned}
$$

Dimensionless form of (10) is given by

$$
\begin{aligned}
& \left(\mathrm{Re}_{w}-\mathrm{Re}_{\infty}\right)^{-1 / 2} \mathrm{Nu}_{x}=-\theta^{\prime}(0), \\
& \left(\mathrm{Re}_{w}-\mathrm{Re}_{\infty}\right)^{-1 / 2} \mathrm{Sh}_{x}=-\phi^{\prime}(0),
\end{aligned}
$$

where the Reynolds numbers are defined as

$$
\operatorname{Re}_{w}=\frac{u_{w} x}{v}, \quad \operatorname{Re}_{\infty}=\frac{u_{\infty} x}{v} .
$$

\section{Results and Discussion}

Here the velocity, temperature, and concentration profiles are analyzed for the velocity ratio $\epsilon$, suction parameter $S$, Brownian motion parameter $N_{b}$, thermophoresis parameter $N_{t}$, and

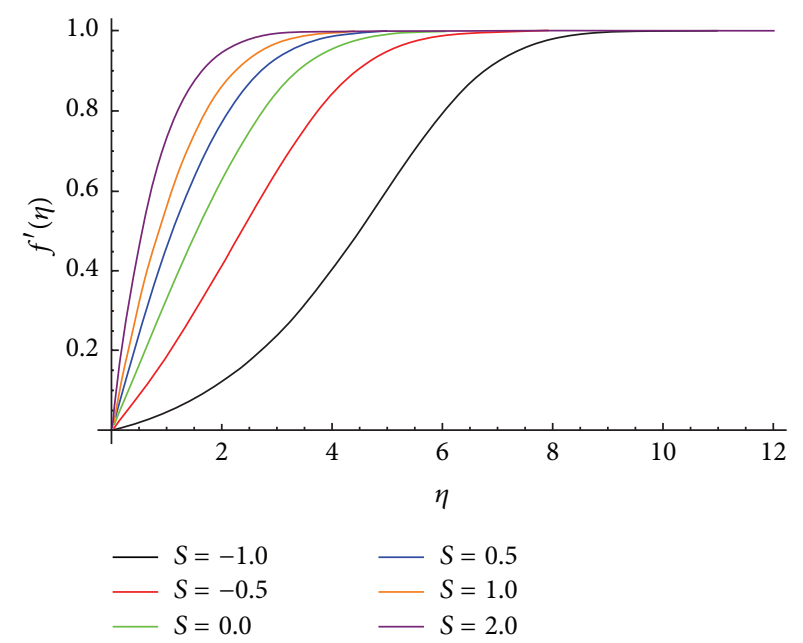

FIGURE 3: Velocity profile $f^{\prime}(\eta)$ for various values of $S$ when $\epsilon=0.0$.

Lewis number Le. Such theme is achieved through the plots of Figures 2-12 which are sketched. Figure 2 describes the effect of $\epsilon$ on $f^{\prime}$. It is found that initially $f^{\prime}$ decreases, but after $\eta=1.0$, it increases when $\epsilon$ decreases. Figures 3 and 4 study the influence of $S$ on $f^{\prime}$ when $\epsilon=0.0$ and $\epsilon=0.3$, respectively. The boundary layer thickness is found to decrease with the increasing values of $S$. Sucking fluid particles through porous wall reduces the growth of the boundary layer. This is quite reliable as the fact that suction causes reduction in the boundary layer thickness. Hence a porous character of wall provides a powerful mechanism for controlling the momentum boundary layer thickness. Influence of parameter $\gamma$ on dimensionless temperature $\theta$ is seen in Figure 5. A gradual increase in $\gamma$ increases the thermal boundary layer thickness. This is expected because the thermal resistance on the hot fluid side is proportional to $h_{f}$. Hence when $\gamma$ increases, the hot fluid side convection decreases and consequently the surface temperature increases. Also for $\alpha \rightarrow \infty$, the result approaches the classical solution for the constant surface temperature. For fixed values of cold fluid properties and free stream velocity, $\alpha$ at any location $x$ is directly proportional to heat transfer coefficient associated with the hot fluid, namely, $h_{f}$. The thermal resistance on the hot fluid side is inversely proportional to $h_{f}$. Thus when $\alpha$ increases, then hot fluid side convection resistance decreases and, consequently, the surface temperature increases [21]. Figure 6 elucidates the effects of $S$ on $\theta$. Temperature field $\theta$ decreases when $S$ increases. The thermal boundary layer thickness also decreases by increasing $S$. Effects of thermophoresis parameter $N_{t}$ and Brownian motion parameter $N_{b}$ on the temperature $\theta$ are shown in Figures 7 and 8. An appreciable increase in the temperature and thermal boundary layer thickness is noticed with an increase in $N_{t}$ and $N_{b}$. The Brownian motion of nanoparticles contributes to thermal conduction enhancement and hence both the temperature and thermal boundary layer thickness increase. It is also noticed that such increase is larger in the case of $N_{b}$ when compared with $N_{t}$. Figure 9 illustrates the effect of Lewis number Le on mass fraction field $\phi$. An increase in Le leads to 
TABLE 1: Values of $\theta(0)$ and $-\theta^{\prime}(0)$ for various values of $\gamma$ when $N_{b}=0=N_{t}=S=\epsilon=0.0$.

\begin{tabular}{|c|c|c|c|c|c|c|c|c|}
\hline \multirow{3}{*}{$\gamma$} & \multicolumn{2}{|c|}{$\theta(0)$} & \multicolumn{2}{|c|}{$-\theta^{\prime}(0)$} & \multicolumn{2}{|c|}{$\theta(0)$} & \multicolumn{2}{|c|}{$-\theta^{\prime}(0)$} \\
\hline & \multicolumn{4}{|c|}{$\operatorname{Pr}=0.1$} & \multicolumn{4}{|c|}{$\operatorname{Pr}=10$} \\
\hline & [21] & Present & [21] & Present & [21] & Present & [21] & Present \\
\hline 0.05 & 0.2536 & 0.25362 & 0.0373 & 0.03733 & 0.0643 & 0.06427 & 0.0468 & 0.04683 \\
\hline 0.10 & 0.4046 & 0.40463 & 0.0594 & 0.05941 & 0.1208 & 0.12075 & 0.0879 & 0.08778 \\
\hline 0.20 & 0.5761 & 0.57613 & 0.848 & 0.84821 & 0.2155 & 0.21552 & 0.1569 & 0.15685 \\
\hline 0.40 & 0.7310 & 0.73104 & 0.1076 & 0.10762 & 0.3546 & 0.35458 & 0.2582 & 0.25823 \\
\hline 0.60 & 0.8030 & 0.80306 & 0.1182 & 0.11824 & 0.4518 & 0.45177 & 0.3289 & 0.32877 \\
\hline 0.80 & 0.8446 & 0.84463 & 0.1243 & 0.12434 & 0.5235 & 0.52354 & 0.3812 & 0.38134 \\
\hline 1 & 0.8717 & 0.87172 & 0.1283 & 0.12833 & 0.5787 & 0.57868 & 0.4213 & 0.42125 \\
\hline 5 & 0.9714 & 0.97144 & 0.1430 & 0.14395 & 0.8729 & 0.87289 & 0.6356 & 0.63558 \\
\hline 10 & 0.9855 & 0.98554 & 0.1450 & 0.14597 & 0.9321 & 0.93207 & 0.6787 & 0.67856 \\
\hline 20 & 0.9927 & 0.99275 & 0.1461 & 0.14607 & 0.9649 & 0.96491 & 0.7026 & 0.70246 \\
\hline
\end{tabular}

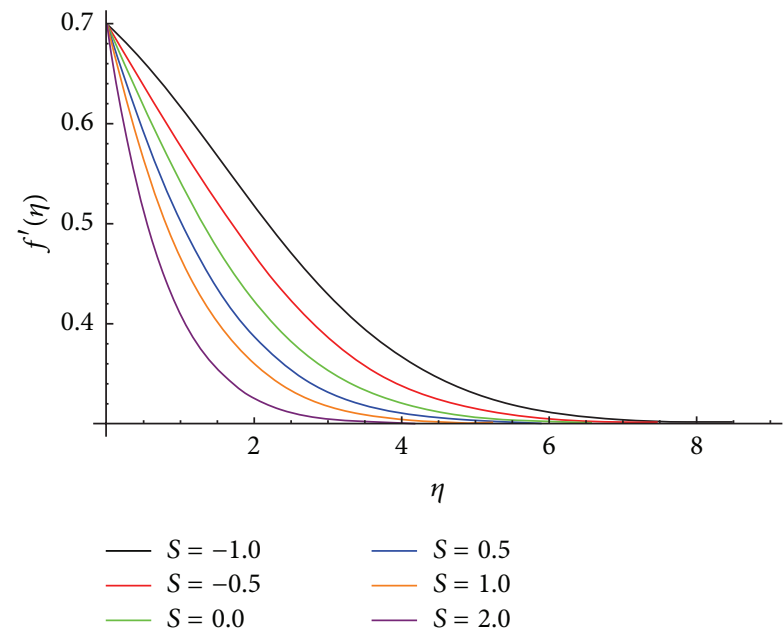

FIGURE 4: Velocity profile $f^{\prime}(\eta)$ for various values of $S$ when $\epsilon=0.0$.

a decrease in molecular diffusivity. Thus increasing the values of Le gradually decreases the concentration of boundary layer. Figures 10 and 11 are plotted to show the effects of thermophoresis parameter $N_{t}$ and Brownian motion parameter $N_{b}$, respectively, on concentration of field $\phi$. It is observed that an increase in $N_{b}$ increases the concentration boundary layer whereas an increase in $N_{t}$ causes a decrease in $\phi$. Figure 12 shows the effect of $\gamma$ on the mass fraction field $\phi$. It is also observed that $N_{t}=0=N_{b}$ corresponds to the case when there is no transport driven by the moment of nanoparticles from the surface to the fluid. Further for $\epsilon=0.0$, our results are in excellent agreement with those presented in [21] (see Table 1).

3.1. Conclusions. An incompressible two-dimensional boundary layer flow of nanofluids past a permeable moving surface with convective boundary conditions is studied numerically. The governing boundary layer equations are converted into highly nonlinear coupled ordinary differential equations using some suitable transformations. The resulting

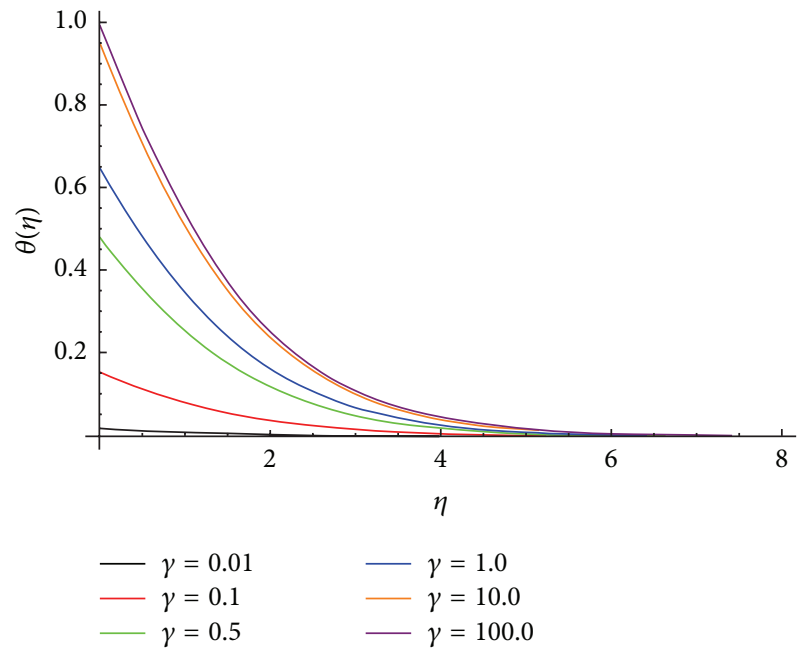

FIgURE 5: Temperature profile $\theta(\eta)$ for various values of $\gamma$ when $\operatorname{Pr}=$ $0.7, S=1.0, N_{t}=N_{b}=0.1$, and $\epsilon=0.3$.

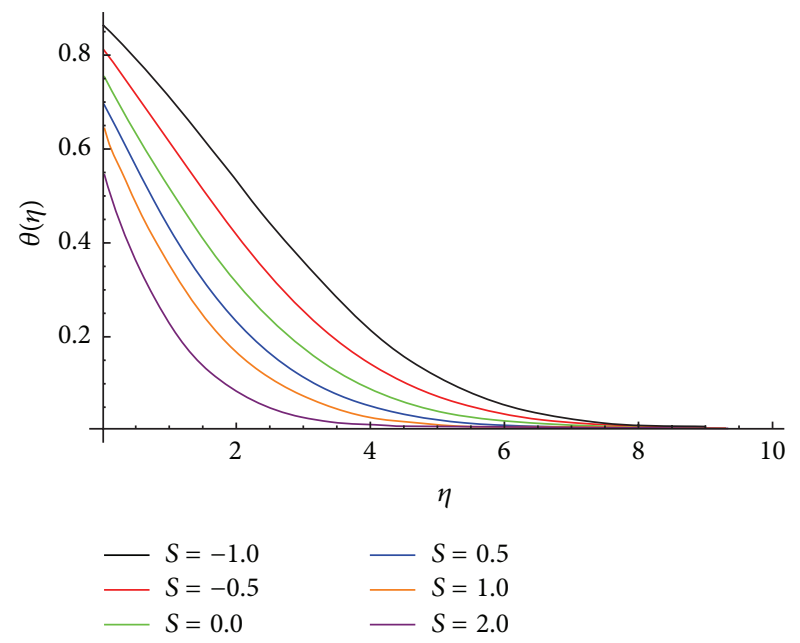

Figure 6: Temperature profile $\theta(\eta)$ for various values of $S$ when $\operatorname{Pr}=0.7, \gamma=1.0, N_{b}=0.2$, Le $=5.0$, and $\epsilon=0.3$. 


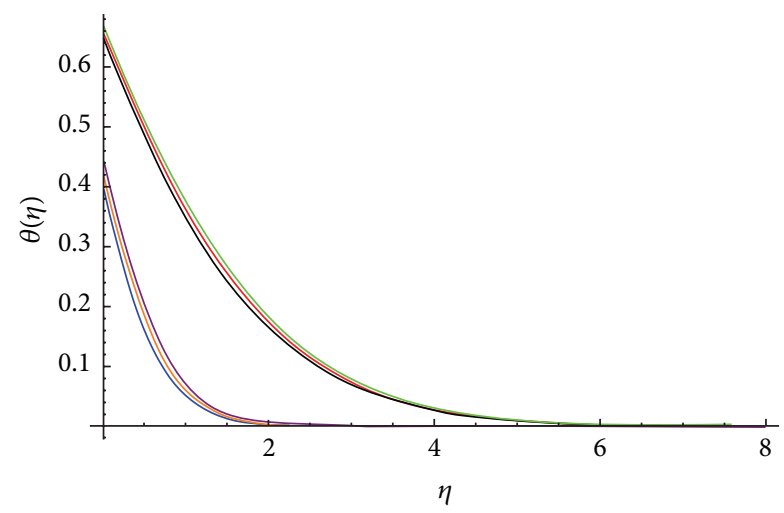

$$
\begin{array}{rlrl}
-N_{t} & =0.1, \operatorname{Pr}=0.7 & -N_{t}=0.1, \operatorname{Pr}=3.0 \\
-N_{t}=0.2, \operatorname{Pr}=3.0 \\
N_{t}=0.1, \operatorname{Pr}=0.7 & -N_{t}=0.3, \operatorname{Pr}=3.0
\end{array}
$$

Figure 7: Temperature profile $\theta(\eta)$ for various values of $N_{t}$ when $\operatorname{Pr}=0.7, \gamma=1.0, N_{b}=0.2$, Le $=5.0$, and $\epsilon=0.3$.

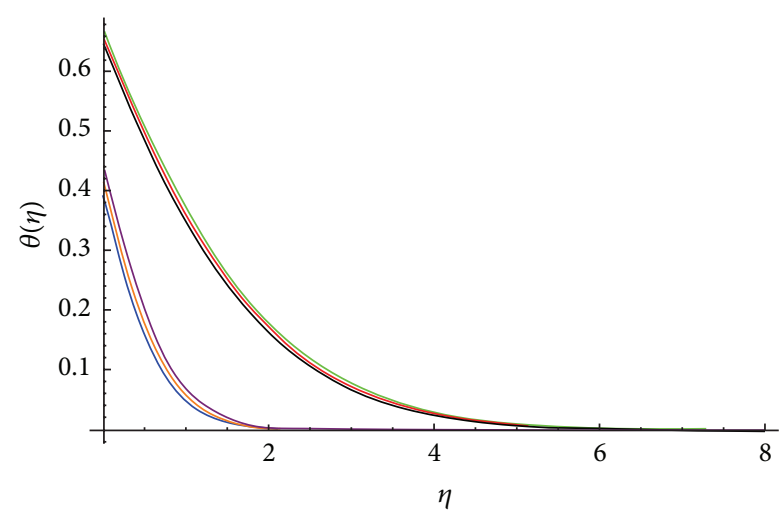

$$
\begin{aligned}
& -N_{b}=0.1, \operatorname{Pr}=0.7 \quad-N_{b}=0.1, \operatorname{Pr}=3.0 \\
& -N_{b}=0.3, \operatorname{Pr}=0.7 \quad-N_{b}=0.3, \operatorname{Pr}=3.0 \\
& -N_{b}=0.5, \operatorname{Pr}=0.7 \quad-N_{b}=0.5, \operatorname{Pr}=3.0
\end{aligned}
$$

FIGURE 8: Temperature profile $\theta(\eta)$ for various values of $N_{b}$ when $\operatorname{Pr}=0.7, \gamma=1.0, N_{t}=0.3$, Le $=5.0$, and $\epsilon=0.3$.

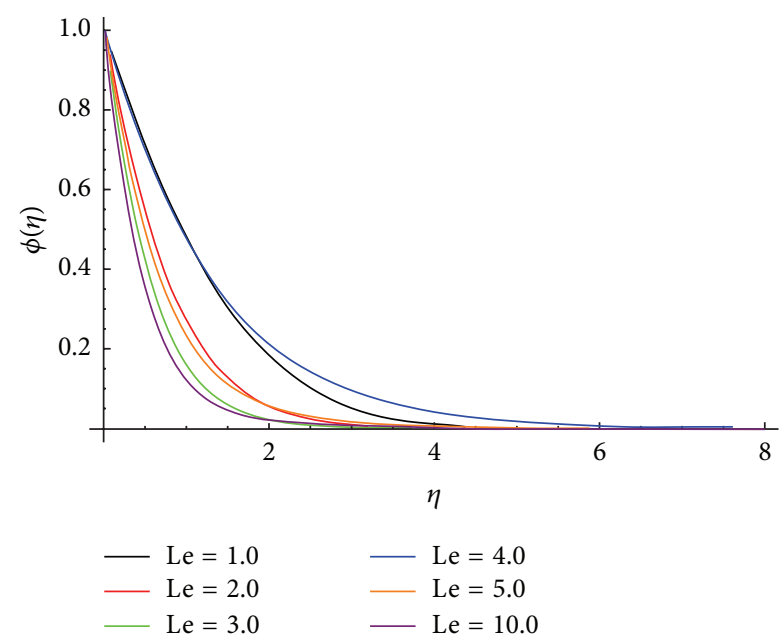

FIGURE 9: Concentration profile $\phi(\eta)$ for various values of Le when $\operatorname{Pr}=0.7, \gamma=1.0, N_{t}=0.3, N_{t}=0.1$, and $\epsilon=0.3$.

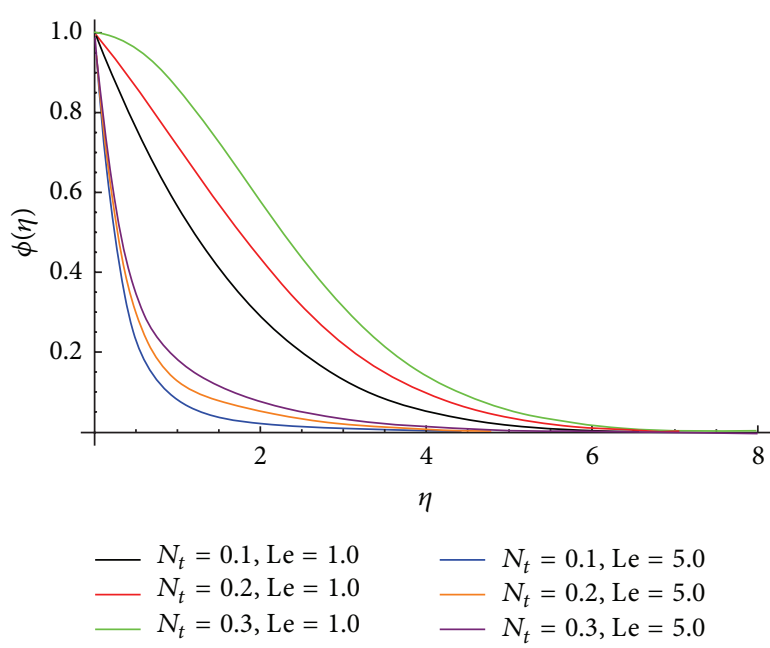

FIGURE 10: Concentration profile $\phi(\eta)$ for various values of $N_{t}$ when $\operatorname{Pr}=0.7, \gamma=1.0, N_{b}=0.3$, and $\epsilon=0.3$.

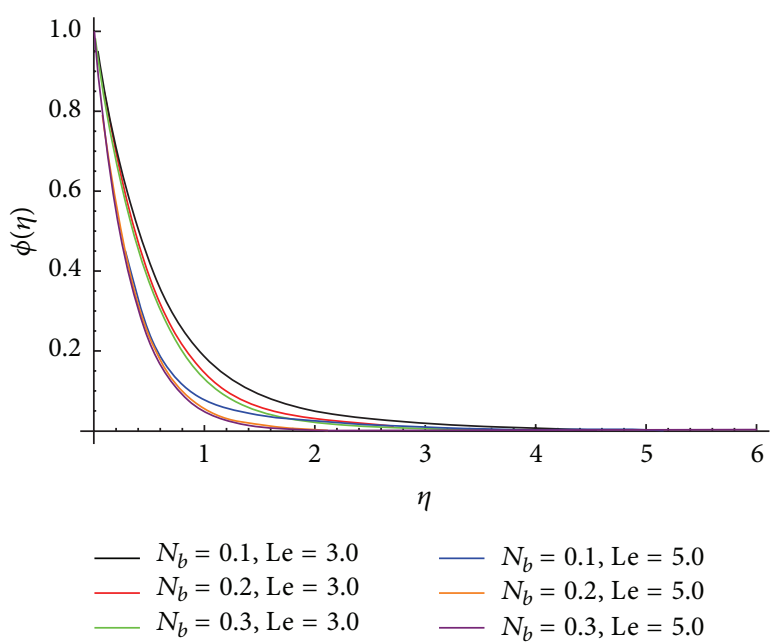

FIGURE 11: Concentration profile $\phi(\eta)$ for various values of $N_{b}$ when $\operatorname{Pr}=0.7, \gamma=1.0, N_{t}=0.3$, and $\epsilon=0.3$.

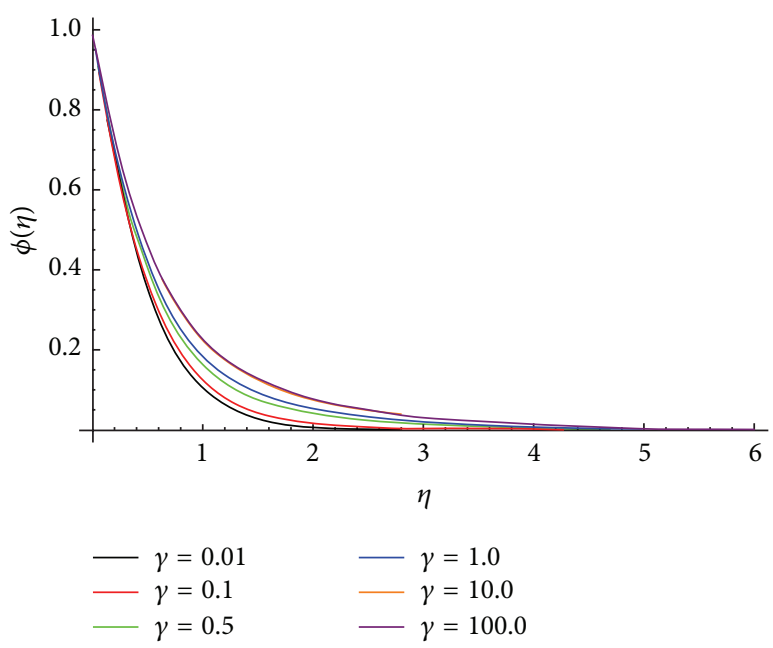

FIGURE 12: Concentration profile $\phi(\eta)$ for various values of $\gamma$ when $\operatorname{Pr}=0.7, N_{b}=0.1, N_{t}=0.3$, and $\epsilon=0.3$. 
equations are solved numerically using shooting technique. Based on the results, the following conclusions are drawn.

(i) $f^{\prime}$ decreases initially, but after $\eta=1.0$, it increases when $\epsilon$ decreases.

(ii) Boundary layer thickness decreases with the increasing values of $S$.

(iii) Porous character of wall provides a powerful mechanism for controlling the momentum of boundary layer thickness.

(iv) As $\gamma$ increases, it increases the thermal boundary layer thickness.

(v) Temperature decreases when $S$ increases.

(vi) Temperature and thermal boundary layer thickness increase with increasing $N_{t}$ and $N_{b}$.

(vii) An increase in $N_{b}$ increases the concentration of boundary layer whereas an increase in $N_{t}$ causes a decrease in $\phi$.

(viii) Results in [21] are found to be special cases of the present work.

\section{References}

[1] S. Choi, "Enhancing thermal conductivity of fluids with nanoparticle," in Developments and Applications of Non-Newtonian Flows, D. A. Siginer and H. P. Wang, Eds., vol. 66, pp. 99-105, ASME, FED, 1995.

[2] A. B. Rosmila, R. Kandasamy, and I. Muhaimin, "Lie symmetry group transformation for MHD natural convection flow of nanofluid over linearly porous stretching sheet in presence of thermal stratification," Applied Mathematics and Mechanics, vol. 33, no. 5, pp. 593-604, 2012.

[3] J. Buongiorno, "Convective transport in nanofluids," Journal of Heat Transfer, vol. 128, no. 3, pp. 240-250, 2006.

[4] P. Rana and R. Bhargava, "Flow and heat transfer of a nanofluid over a nonlinearly stretching sheet: a numerical study," Communications in Nonlinear Science and Numerical Simulation, vol. 17, no. 1, pp. 212-226, 2012.

[5] R. Kandasamy, P. Loganathan, and P. P. Arasu, "Scaling group transformation for MHD boundary-layer flow of a nanofluid past a vertical stretching surface in the presence of suction/ injection," Nuclear Engineering and Design, vol. 241, no. 6, pp. 2053-2059, 2011.

[6] M. A. A. Hamad and M. Ferdows, "Similarity solution of boundary layer stagnation-point flow towards a heated porous stretching sheet saturated with a nanofluid with heat absorption/generation and suction/blowing: a Lie group analysis," Communications in Nonlinear Science and Numerical Simulation, vol. 17, no. 1, pp. 132-140, 2012.

[7] A. Mahdy, "Unsteady mixed convection boundary layer flow and heat transfer of nanofluids due to stretching sheet," Nuclear Engineering and Design, vol. 249, pp. 248-255, 2012.

[8] M. I. Anwar, I. Khan, S. Sharidan, and M. Z. Salleh, "Conjugate effects of heat and mass transfer of nanofluids over a nonlinear stretching sheet," International Journal of Physical Sciences, vol. 7, pp. 4081-4092, 2012.

[9] M. I. Anwar, I. Khan, M. Z. Salleh, A. Hasnain, and S. Sharidan, "Magnetohydrodynamic effects on stagnation-point flow of
Nanofluids towards a non-linear stretching sheet," Wulfenia Journal, vol. 19, pp. 367-383, 2012.

[10] W. A. Khan and I. Pop, "Boundary layer flow past a stretching surface in a porous medium saturated by a nanofluid: Brinkman-Forchheimer model," PLoS ONE, vol. 7, no. 10, Article ID e47031, 2012.

[11] O. D. Makainde, W. A. Khan, and Z. H. Khan, "Buoyancy effects on MHD stagnation point flow and heat transfer of a nanofluid past a convectively heated stretching/shrinking sheet," International Journal of Heat and Mass Transfer, vol. 62, pp. 526-533, 2013.

[12] S. Nadeem, R. U. Haq, and Z. H. Khan, "Numerical solution of non-Newtonian nanofluid flow over a stretching sheet," Applied Nanoscience 07/s13204-013-0235-8.

[13] A. Aziz and W. A. Khan, "Natural convective boundary layer flow of a nanofluid past a convectively heated vertical plate," International Journal of Thermal Sciences, vol. 52, no. 1, pp. 8390, 2012.

[14] S. Nadeem, R. Mehmood, and N. S. Akbar, "Non-orthogonal stagnation point flow of a nano non-Newtonian fluid towards a stretching surface with heat transfer," International Journal of Heat and Mass Transfer, vol. 57, pp. 679-689, 2013.

[15] W. A. Khan, A. Aziz, and N. Uddin, "Buongiorno model for nanofluid blasius flow with surface heat and mass fluxes," Journal of Thermophysics and Heat Transfer, vol. 27, no. 1, pp. 134-141, 2013.

[16] B. C. Sakiadis, "Boundary-layer behavior on continuous solid surfaces: I. Boundary-layer equations for two-dimensional and axisymmetric flow, AIChE Journal, vol. 7, pp. 26-28, 1961.

[17] F. K. Tsou, E. M. Sparrow, and R. J. Goldstein, "Flow and heat transfer in the boundary layer on a continuous moving surface," International Journal of Heat and Mass Transfer, vol. 10, no. 2, pp. 219-235, 1967.

[18] T. A. Abdelhafez, "Skin friction and heat transfer on a continuous flat surface moving in a parallel free stream," International Journal of Heat and Mass Transfer, vol. 28, no. 6, pp. 1234-1237, 1985.

[19] N. Afzal, A. Badaruddin, and A. A. Elgarvi, "Momentum and heat transport on a continuous flat surface moving in a parallel stream," International Journal of Heat and Mass Transfer, vol. 36, no. 13, pp. 3399-3403, 1993.

[20] A. Ishak, R. Nazar, and I. Pop, “The effects of transpiration on the flow and heat transfer over a moving permeable surface in a parallel stream," Chemical Engineering Journal, vol. 148, no. 1, pp. 63-67, 2009.

[21] A. Aziz, "A similarity solution for laminar thermal boundary layer over a flat plate with a convective surface boundary condition," Communications in Nonlinear Science and Numerical Simulation, vol. 14, no. 4, pp. 1064-1068, 2009.

[22] T. Hayat, S. A. Shehzad, M. Qasim, and S. Obaidat, "Steady flow of Maxwell fluid with convective boundary conditions," Zeitschrift fur Naturforschung A, vol. 66, no. 6-7, pp. 417-422, 2011.

[23] O. D. Makinde, "Thermal stability of a reactive viscous flow through a porous-saturated channel with convective boundary conditions," Applied Thermal Engineering, vol. 29, no. 8-9, pp. 1773-1777, 2009.

[24] O. D. Makinde and A. Aziz, "MHD mixed convection from a vertical plate embedded in a porous medium with a convective boundary condition," International Journal of Thermal Sciences, vol. 49, no. 9, pp. 1813-1820, 2010. 
[25] W. A. Khan and R. S. R. Gorla, "Mixed convection of water at $4^{\circ} \mathrm{C}$ along a wedge with a convective boundary condition in a porous medium," Special Topics and Reviews in Porous Media, vol. 2, no. 3, pp. 227-236, 2011.

[26] M. A. A. Hamad, M. J. Uddin, and A. I. M. Ismail, "Investigation of combined heat and mass transfer by Lie group analysis with variable diffusivity taking into account hydrodynamic slip and thermal convective boundary conditions," International Journal of Heat and Mass Transfer, vol. 55, no. 4, pp. 1355-1362, 2012.

[27] N. A. Yacob, A. Ishak, I. Pop, and K. Vajravelu, "Boundary layer flow past a stretching/shrinking surface beneath an external uniform shear flow with a convective surface boundary condition in a nanofluid," Nanoscale Research Letters, vol. 6, article 314, 2011.

[28] O. D. Makinde, T. Chinyoka, and L. Rundora, "Unsteady flow of a reactive variable viscosity non-Newtonian fluid through a porous saturated medium with asymmetric convective boundary conditions," Computers \& Mathematics with Applications, vol. 62, no. 9, pp. 3343-3352, 2011.

[29] R. C. Bataller, "Radiation effects for the Blasius and Sakiadis flows with a convective surface boundary condition," Applied Mathematics and Computation, vol. 206, no. 2, pp. 832-840, 2008. 


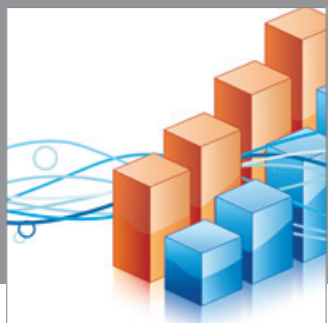

Advances in

Operations Research

mansans

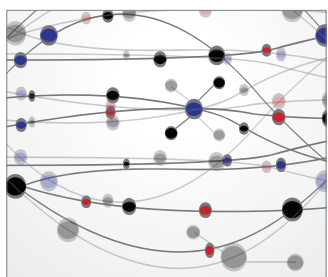

The Scientific World Journal
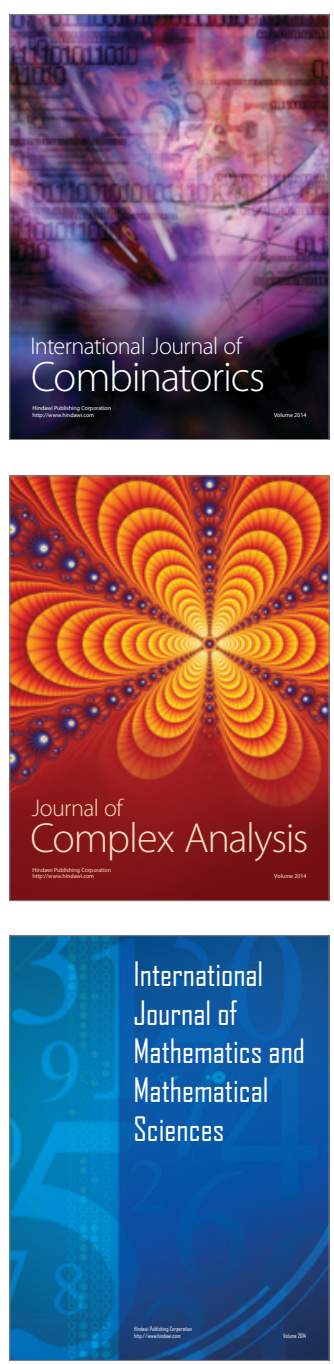
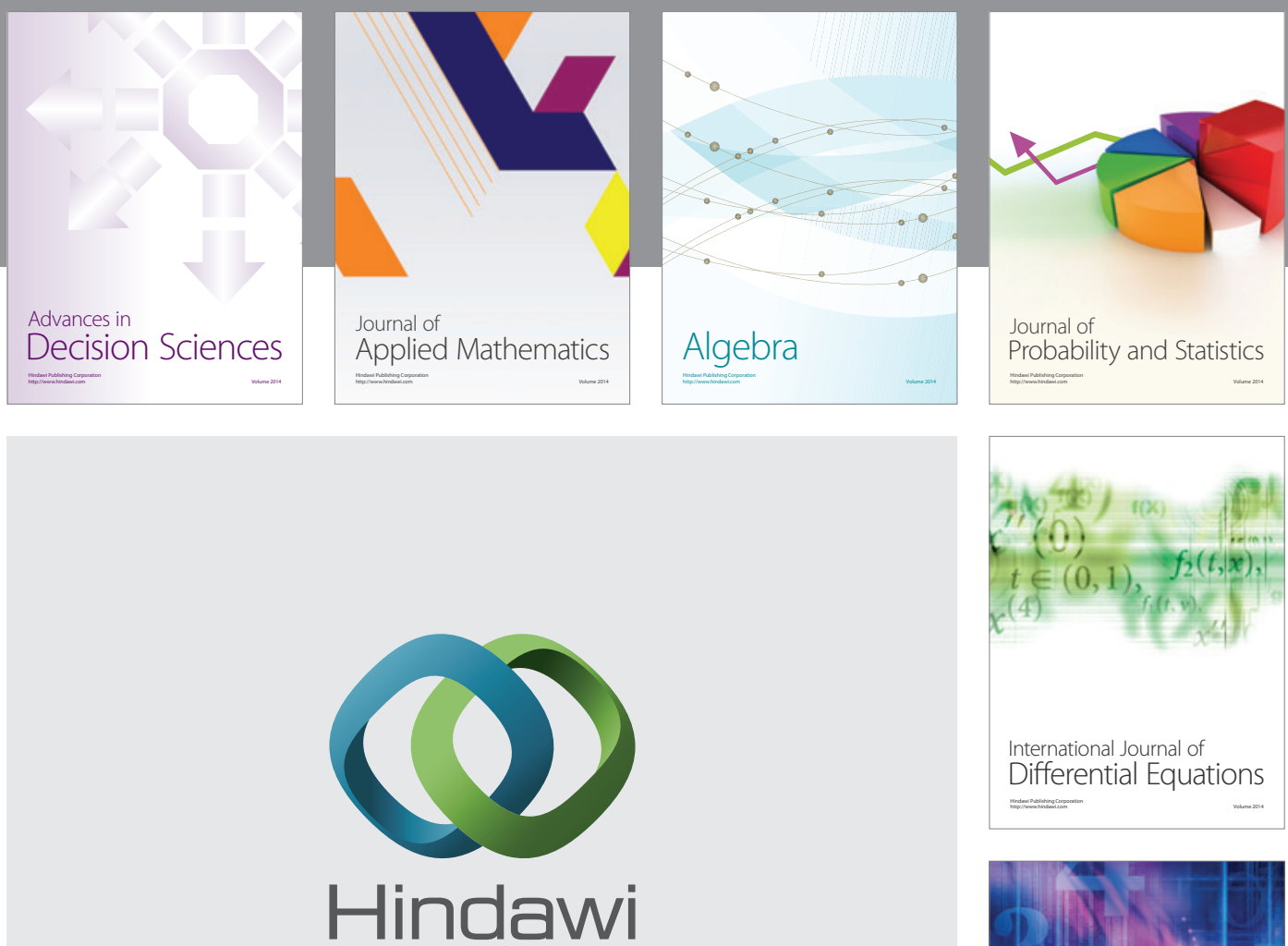

Submit your manuscripts at http://www.hindawi.com
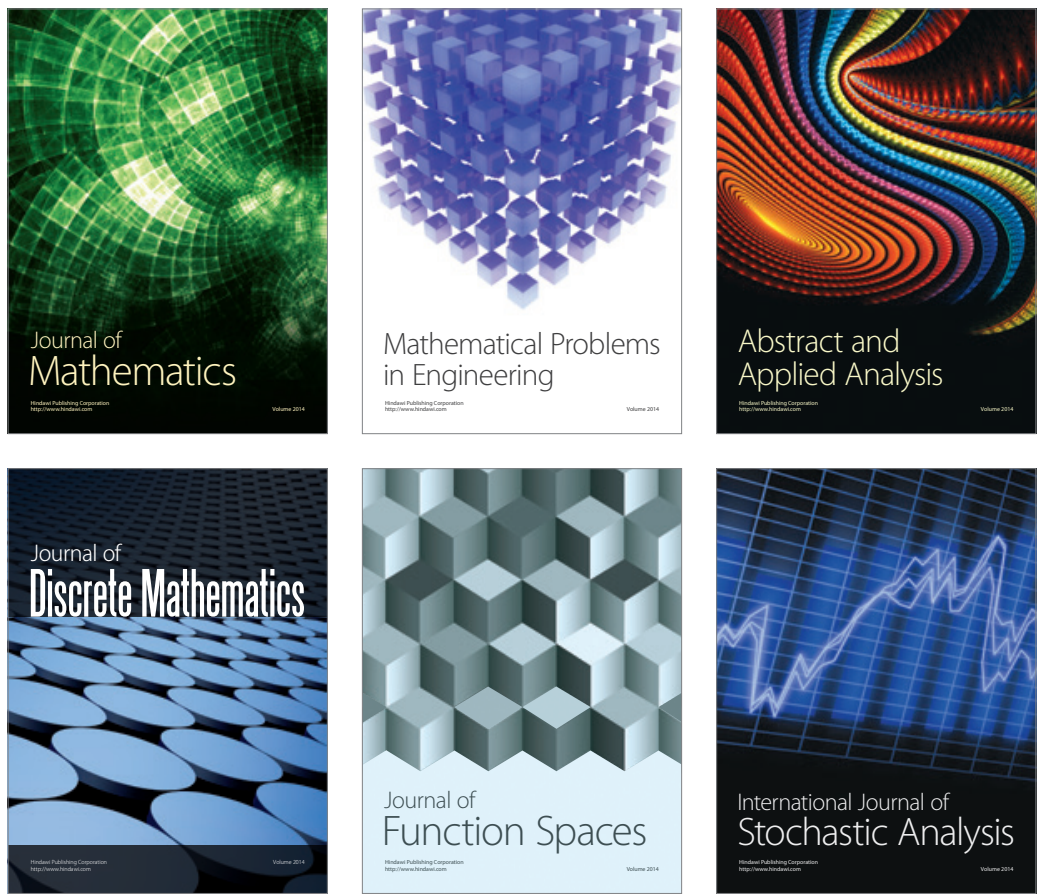

Journal of

Function Spaces

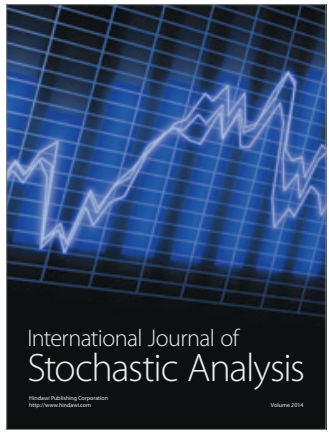

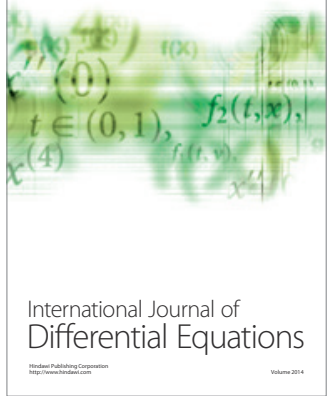
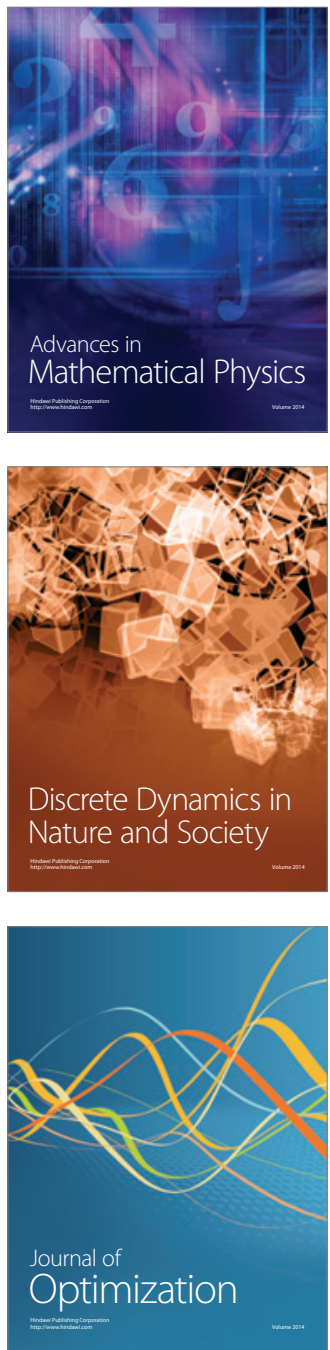